A - Research concept and design

B - Collection and/or assembly of data

$\mathrm{C}$ - Data analysis and interpretation

D - Writing the article

E - Critical revision of the article

F - Final approval of article

\section{Effect of spinal manipulation on cardiopulmonary parameters in patients with functional scoliosis: A randomised controlled pilot trial}

\author{
Charu Gera ${ }^{1, A-F^{*}}$ (D), Manoj Malik ${ }^{2, A-F}$ (iD \\ ${ }^{1}$ Guru Jambheshwar University of Science and Technology, Hisar, Haryana, \\ India \\ ${ }^{2}$ Department of Physiotherapy, Guru Jambheshwar University of Science \\ and Technology, Hisar, Haryana, India
}

*Correspondence: Charu Gera; Guru Jambheshwar University of Science and Technology, Hisar, Haryana, India; email: charugera321@gmail.com

\title{
Abstract
}

Introduction: This pilot trial reports the findings of randomized controlled trial (RCT) investigating the initial estimates of the effects of spinal manipulations on systolic blood pressure (SBP), diastolic blood pressure (DBP) and oxygen saturation $(\mathrm{SpO} 2)$ in patients suffering from functional scoliosis as well as determine the feasibility and acceptability of spinal manipulation in the management of functional scoliosis for full trial and to obtain pilot data to perform sample size calculation for full trial.

Material and methods: A Single-blinded trial was done in accordance with CONSORT guidelines 2010. Thirty patients with functional scoliosis with 'Intention to treat Analysis' were recruited. The participants were randomly allocated into experimental $(n=15)$ and control group $(n=15)$. Experimental group received spinal manipulations with Grade 5 high velocity thrust and Schroth exercise. Control group received only Schroth exercise. Feasibility and acceptability were assessed by number of participants completing the baseline and post-intervention data of treatment process.

Results: Experimental group showed a statistically significant improvement in $\mathrm{SBP}(\mathrm{MD}=10.73 ; 95 \% \mathrm{CI}=4.63$, 7.49), $\mathrm{DBP}(\mathrm{MD}=10.13 ; 95 \% \mathrm{CI}=5.80,8.06)$ and $\mathrm{SpO} 2(\mathrm{MD}=-7.2 ; 95 \% \mathrm{CI}=-5.55,-3.51)$ as compared to control group in terms of SBP $(\mathrm{MD}=4.66 ; 95 \% \mathrm{CI}=3.63,5.50), \mathrm{DBP}(\mathrm{MD}=3.2 ; 95 \% \mathrm{CI}=3.20,4.01)$ and $\mathrm{SpO} 2(\mathrm{MD}=-2.7$; $95 \% \mathrm{CI}=-3.55,-2.50)$. Results suggested that outcome measures were feasible and acceptable.

Conclusions: This pilot study is feasible and acceptable to do a fully powered randomised controlled trial. This study also concluded that spinal manipulations combined with Schroth exercises may significantly reduce the blood pressures and improve the oxygen saturation levels.

\section{Keywords: blood pressure, manual therapy, functional scoliosis, randomised controlled pilot} trial, Schroth exercises

\section{Introduction}

Correct posture is one of the most crucial aspects of healthy body functioning and contribute to a good appearance. The proper and correct alignment of body segments also promotes the efficiency of movement, activities, and endurance. Good posture also maintains the balance between the musculature, bones and joints [1]. It is evident that poor or incorrect posture in one part of the body can result in an imbalance of adjacent

This is an Open Access journal, all articles are distributed under the terms of the Creative Commons Attribution-NonCommercial-ShareAlike 4.0 International (CC BY-NC-SA 4.0). License (http://creativecommons. org/licenses/by-nc-sa/4.0/). 
parts of the body as a compensatory mechanism. These imperfect interrelations affect the position, musculature and functioning of vital organs. This in turn makes joint movement more difficult and may cause pain and fatigue $[1,2]$. Poor posture can produce an excessive strain on adjacent structures and less-efficient balance of the body [3]. The most common postural malalignments that affect the human body are forward head posture, rounded shoulders, cervical malalignments, kyphosis, lordosis and scoliosis [3].

According to existing research, the most prevalent postural spinal disorder is adolescent idiopathic scoliosis (AIS), with $0.4-5.2 \%$ prevalence globally worldwide [4]. The prevalence rate of scoliosis in the elderly population has been reported to be more than $60 \%[5,6]$. "Non-structural" or "functional" scoliosis is considered to be temporary and reversible lateral curvatures that occur naturally in response to abnormal posture. It results in non-structural, temporary and compensatory changes in spinal curvature [7]. The most common risk factors that result in functional scoliosis include abnormal postural habits, altered biomechanics, muscular imbalances, joint laxity, and factors such as work stress, pressure and emotional burden [7]. Scoliosis may also occur due to leg length discrepancy [8]. Scoliosis has many negative effects on the human body, such as spinal malalignments, joint imbalance and muscle imbalance (such as tightening and weakening) [9]. It can also cause excessive strain on the musculature, joints, tendons and bones of the spine, which can lead to pain, fatigue and deformation of spinal curvature [7]. Any changes in the thoracic spine, along with the thoracic cage, can affect the functioning of the lungs and heart. The literature suggests a strong correlation of scoliosis with the cardiopulmonary system $[10,11]$ such as hypertension, [12] migraine [13] and psychosomatic symptoms like stress, anxiety and depression [9,14-16]. Therefore, it can be considered a serious condition that affects the quality of life.

Hence, it is very important to recognize the clinical symptoms and treat these postural malalignments with the appropriate treatment strategies to improve the quality of life. According to the International Society on Scoliosis Orthopaedic and Rehabilitation Treatment (SOSORT) guidelines for the management of scoliosis, a multidisciplinary approach including spinal exercises, spinal manipulations, posture correction exercises and respiratory exercises is recommended [17]. Postural correction exercises (such as the Schroth protocol) contribute to an increase in the depth of breath and in lung capacity. Posture correction exercises also positively affect the nervous system by stimulating the motor and sensory areas of the brain. Such exercises can also help improve the cardio-respiratory system [18]. Postural exercises, yoga and resistance band exercises can correct the abnormal postural as well as reduce depression and anxiety associated with abnormal postural [19-21].

Therefore, the purpose of the present randomized controlled pilot trial was to evaluate a preliminary examination of the effects of spinal manipulations on systolic blood pressure (SBP), diastolic blood pressure (DBP) and oxygen saturation (SpO2) in patients suffering from functional scoliosis, as well as to determine the feasibility and acceptability of spinal manipulation in the management of functional scoliosis for a full trial and to obtain pilot data for sample size calculation of the full trial.

\section{Material and methods}

A single-blinded randomized controlled pilot trial was conducted to assess the effects of spinal manipulations and Schroth exercise protocol on SBP, DBP and $\mathrm{SpO} 2$ in patients suffering from functional scoliosis. This pilot trial was conducted in accordance with the Consolidated Standards of Reporting Trials (CONSORT) guidelines 2010. The study protocol was approved by the Institute Ethics Committee (IEC), vide letter no. PTY/2019/1014, dated 11.09.19. This trial is registered with the Clinical Trial Registry of India (Registration no. CTRI/2020/02/023221.

\section{Participants}

The participants were randomly allocated into two groups: experimental group $(\mathrm{n}=15)$ and control group $(n=15)$. This allocation was done using the computer-generated random number table method. The assessment was performed at baseline and after 3 weeks. All patients received an explanation of the trial in the local vernacular language and written informed consent was taken from them according to the Declaration of Helsinki, 2013. The intervention was started after randomization. Subjects were recruited from the Outpatient Department of the Health Center of the university and private clinics. Thirty patients with functional scoliosis were recruited in this trial. Patients in the 18-50 age group with functional scoliosis with hypertension were included. The exclusion criteria were as follows: spine surgery in the last 6 months, osteoporosis, recent injuries like fractures, grade 3 ligament injuries, vascular disorders, hypermobility and Red flag signs (severe pain, fever, swelling, neurological symptoms, etc). Individuals with functional scoliosis fulfilling the selection criteria were chosen for the study. Informed consent was taken from the participants. 


\section{Procedure}

After the baseline clinical evaluation, patients fulfilling the selection criteria were invited to participate in the trial. Subjects were screened for functional scoliosis by subjective data, observation, postural assessment (using a plumb line), palpation and special tests (Adam's forward bend test) [22]. Pre-intervention data was collected for the readings of SBP, DBP and $\mathrm{SpO} 2$. Before the commencement of intervention, readings for blood pressure and oxygen saturation were obtained from the participants by a skilled and experienced doctor in the department twice daily (i.e. 9 am after the meal and $5 \mathrm{pm}$ on the same day). This procedure used a manual cuff with a mercury sphygmomanometer along with a stethoscope and pulse oximeter. The reliability and validity of the pulse oximeter is excellent i.e. 0.93 [23]. The reliability and validity of the sphygmomanometer is good i.e. 0.90 [24]. Its margin of error was taken as $5 \%$ to $15 \%$. During treatment patients were instructed to avoid any medicine that might affect the outcome.

The treatment protocol for the experimental group included spinal manipulations with Grade 5 highvelocity thrust and the Schroth exercise protocol. The intervention was provided by a trained physiotherapist. The Schroth exercise protocol consists of sensorimotor training, postural exercises \& breathing exercises, general strengthening exercises to strengthen the muscles and tone of the spine. It includes pelvic tilts, cat-camel, double leg abdominal press, single leg balance, good sitting and standing posture [25]. Treatment was administered for three weeks, thrice weekly. After three weeks, readings of blood pressure and oxygen saturation from the participants were again recorded twice (i.e. 9 am after the meal and $5 \mathrm{pm}$ on the same day). Two readings were taken and the average of these was taken as the final reading. The Schroth exercise protocol was given to the participants of the control group. They were instructed to continue their exercises for 3 weeks. Pre- and post-data was obtained for evaluating any statistically significant change.

\section{Outcome measures}

Outcome measures were blood pressure (SBP and $\mathrm{DBP}$ ) and $\mathrm{SpO} 2$.

\section{Measures to assess Feasibility and Acceptability}

The feasibility and acceptability of the main trial was the primary objective of the pilot study. The feasibility of recruitment rate was assessed by the number of patients recruited in the study according to eligibility criteria, the retention rate was assessed by a number of patients who completed the trial and the adherence rate was assessed by the number of intervention sessions attended by patients and maintaining their presence. The feasibility and acceptability of outcome measures were assessed by the number of participants completing the baseline and post-intervention data of the treatment process. The following were considered as feasibility criteria for the main trial: $70 \%$ participant recruitment, $90 \%$ of participants completing the pilot trial and $95 \%$ of participants giving post-intervention as well as follow-up data with at least $75 \%$ of participants attending all treatment sessions [26].

\section{Working Alliance}

To evaluate the Patient-Therapist relationship, the Working Alliance Inventory-short form was used during post-treatment [27].

\section{Statistical analysis}

Descriptive (mean \pm standard deviation) data were analyzed before and after the intervention. Baseline characteristics were analyzed among the groups to assess the quality of randomization. Between-group differences were evaluated by using an independent t-test. Statistical analysis was performed by using IBM SPSS statistics software 21. The alpha level of significance was set at 0.05 . The $95 \%$ confidence interval was also calculated. This was a pilot study, and with an aim of obtaining data for sample size calculation so as to plan future confirmatory trials. Furthermore, we plan to increase the subjects by $10 \%$, so that the loss in the form of any drop-outs could be compensated.

\section{Results}

Thirty patients with functional scoliosis and hypertension were randomized into experimental $(n=15)$ and control groups $(n=15)$. Intention to treat analysis was performed. One subject dropped out of the control group and his post-intervention data were obtained. This is explained in the CONSORT flow diagram (Fig. 1).

Out of the 30 patients, there were 8 males and 22 females. The baseline characteristics were similar between the two groups (Tab. 1).

Data normality was evaluated through Kolmogorov-Smirnov (K-S) test and Lilliefors corrected K-S test. The results showed that the data were normally distributed.

Between groups analysis were done by independent t-test (Tab. 2.). The results show a statistically significant improvement in the experimental group as compared to the control group in SBP, DBP and SpO2.

Feasibility of outcome measures: All the participants were provided baseline as well as post-intervention data except one drop-out from the control group. 


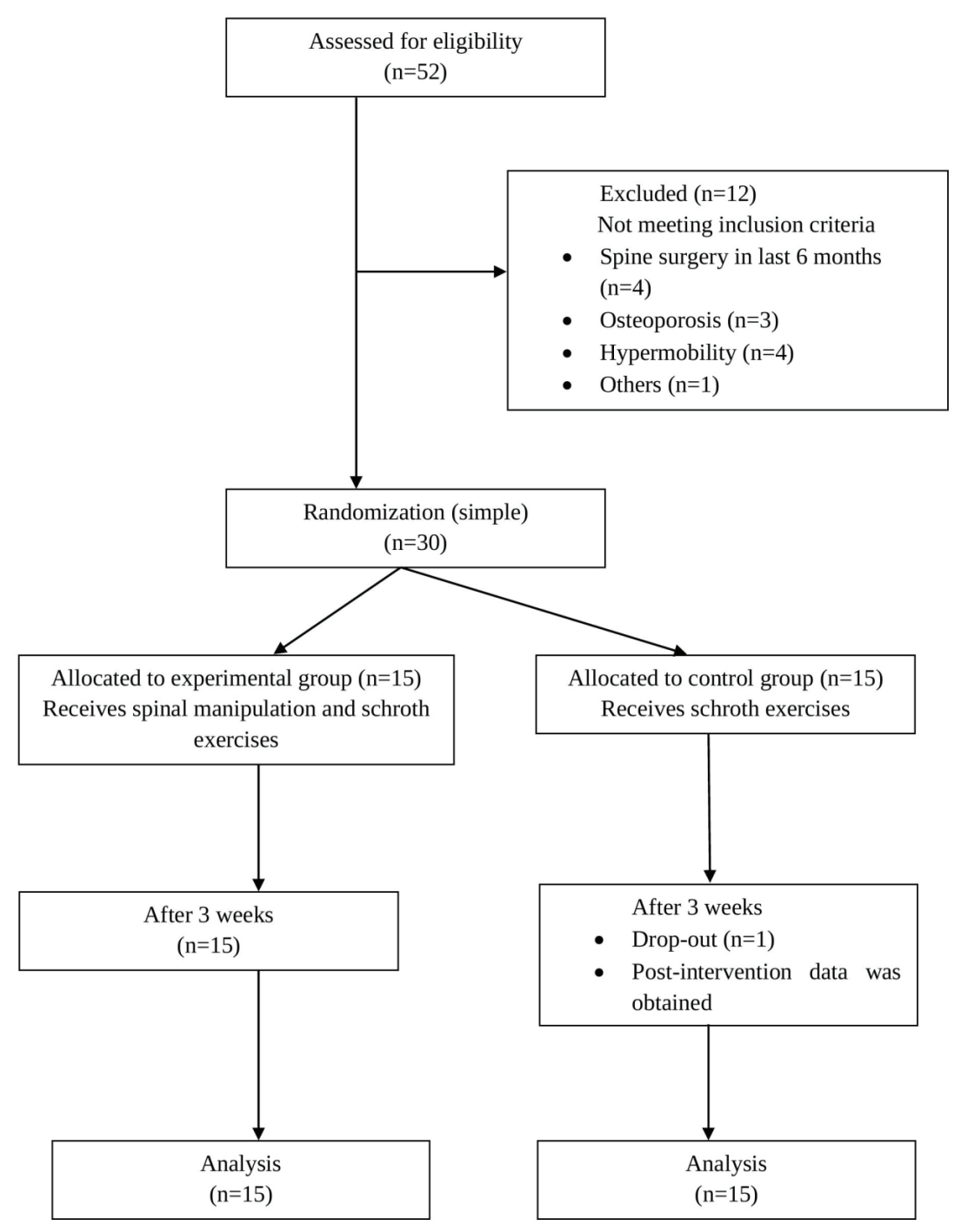

Fig. 1. CONSORT flow diagram

Tab. 1. Participant's baseline characteristics

\begin{tabular}{lccc}
\hline Variable & \multicolumn{3}{c}{ Group } \\
\hline & Experimental (Mean \pm Standard Deviation) & Control (Mean \pm Standard Deviation) & p value \\
\hline Age & $30.13 \pm 3.70$ & $31.3 \pm 4.80$ & 0.45 \\
$\mathrm{SBP}$ & $135.80 \pm 4.82$ & $134.47 \pm 6.03$ & 0.50 \\
$\mathrm{DBP}$ & $97.93 \pm 5.37$ & $99.86 \pm 6.34$ & 0.37 \\
$\mathrm{SpO}_{2}$ & $86.20 \pm 3.80$ & $88.00 \pm 4.20$ & 0.23 \\
\hline
\end{tabular}

DBP - diastolic blood pressure; SBP - systolic blood pressure; SpO2 - oxygen saturation.

Retention rate: At the end of the study, 30/30 (100\%) completed the last week assessment.

Adherence rate: 29 out of 30 participants (96.67\%) completed all the visits.
Acceptability: Most of the patients were satisfied with the spinal manipulation treatment and Schroth exercises except for one participant from the control group. 
Tab. 2. Independent t-test (between the groups)

\begin{tabular}{|c|c|c|c|c|c|c|c|}
\hline \multirow{2}{*}{\multicolumn{2}{|c|}{ Outcome measures }} & \multirow{3}{*}{$\frac{\mathrm{N}}{15}$} & \multirow{3}{*}{$\begin{array}{l}\text { Mean } \\
\text { Comparison } \\
10.73\end{array}$} & \multirow{3}{*}{$\begin{array}{l}\begin{array}{l}\text { Standard } \\
\text { deviation }(\mathrm{SD})\end{array} \\
1.57\end{array}$} & \multicolumn{2}{|c|}{ 95\% Confidence Interval of the Difference } & \multirow{3}{*}{$\begin{array}{l}\begin{array}{l}\text { Sig. } \\
\text { (2-tailed) }\end{array} \\
0.000\end{array}$} \\
\hline & & & & & \multirow{2}{*}{$\begin{array}{l}\text { Lower } \\
4.63\end{array}$} & \multirow{2}{*}{$\begin{array}{l}\text { Upper } \\
7.49\end{array}$} & \\
\hline SBP & Experimental & & & & & & \\
\hline SBP & Control & 15 & 4.66 & 2.19 & 3.63 & 5.50 & 0.000 \\
\hline \multirow{2}{*}{ DBP } & Experimental & 15 & 10.13 & 1.55 & 5.80 & 8.06 & 0.000 \\
\hline & Control & 15 & 3.20 & 1.47 & 3.20 & 4.01 & 0.000 \\
\hline \multirow{2}{*}{$\mathrm{SpO} 2$} & Experimental & 15 & -7.26 & 1.22 & -5.55 & -3.51 & 0.000 \\
\hline & Control & 15 & -2.73 & 1.48 & -3.55 & -2.50 & 0.000 \\
\hline
\end{tabular}

$\mathrm{DBP}$ - diastolic blood pressure; SBP - systolic blood pressure; SpO2 - oxygen saturation.

\section{Discussion}

The primary objective of this trial was to investigate the effect of spinal manipulations and Schroth exercise protocol on SBP, DBP and SpO2 in functional scoliosis. To evaluate this effect, spinal manipulations and the Schroth exercise protocol were given to the patients of the experimental group, while the Schroth exercise protocol alone was given to the patients from the control group. We monitored SBP, DBP and SpO2 before and after the treatment in both experimental and control groups for 3 weeks. The data obtained were compared and analyzed using an independent t-test. There was a statistically significant improvement in the SBP, DBP and $\mathrm{SpO} 2$ of patients in the experimental group as compared to the control group.

This trial focuses on functional scoliosis and its negative effects on SBP, DBP and SpO2. Functional scoliosis is considered as temporary and reversible lateral curvatures that occur naturally in response to abnormal posture or leg length discrepancy. Functional scoliosis results in non-structural, temporary and compensatory changes in spinal curvature $[7,8]$. Hence, it is referred to as structurally normal. Adolescent idiopathic scoliosis (AIS) and functional scoliosis are related, if they both have non-structural curves i.e. a Cobb's angle greater than $10^{\circ}$ and less than $25^{\circ}$. Therefore, it can be treated with conservative management [7]. The appearance of the curve can be affected by body positioning during activities like sitting and standing. Therefore, the proper assessment was done to avoid any positional error during an examination. A spinal curve can also be influenced by X-ray positioning. Evidence also showed that there were changes in the Cobb's angle measurement during radiographs [28]. Further evidence also suggests that faulty and malaligned posture of the thoracic spine and lumbar spine affects blood flow and the diameter of the vertebral artery. Moreover, a decrease in brain blood flow has been strongly linked to hypertension and depression [29]. Changes in the thoracic spine (scoliosis) also result in respiratory and cardiovascular complications [12]. If left untreated, it may cause low oxygen consumption [18].

This trial confirms that spinal manipulations combined with Schroth exercises are effective in decreasing blood pressure. Hence, posture correction by using spinal manipulations may be helpful to manage hypertension and its associated complications [12]. Evidence also suggests that postural exercises, yoga and aerobic exercises may also influence blood pressure. These interventions can increase blood circulation to the heart, lung and brain, thus significantly reducing blood pressure [30]. Research suggests that the use of manipulations, chiropractor techniques and massage promotes relaxation, and reduces stress, depression and anxiety, as well as relieving tense muscles and increasing blood flow to specific areas of the body. Hence, these may result in an overall reduction in mental stress and high blood pressure [31]. All these postural techniques are important in improving psychological stress and high blood pressure. Postural correction exercises also improve oxygen consumption, as they result in an increase in the depth of breath and an increase in lung capacity [18]. The experimental group showed a statistically significant improvement in SBP $(10.73 \pm 1.57)$ and DBP $(10.13 \pm 1.55)$. A meaningful and worthwhile change can be assessed by using minimum clinically important difference (MCID) as a threshold value [32-34]. The commonly accepted MCID for blood pressure is 8 [35], while our trial reported an improvement of 10.13. The experimental group also showed a statistically significant improvement in $\mathrm{SpO} 2$ in terms of the mean change $(-7.27 \pm 1.22)$. The commonly accepted MCID for $\mathrm{SpO} 2$ is 4 [34], while our trial reported a mean improvement of 7.27. Hence, the results for SBP, DBP and $\mathrm{SpO} 2$ were clinically significant. 
This trial has significant clinical and practical implications. Functional scoliosis and hypertension can be one of the most common and serious concerns among the general population. The lifetime risk of developing high blood pressure or hypertension is approximately $90 \%$. This may lead to serious complications such as hemorrhage, stroke, heart attack, breathing problems and so on [12]. Patients are also dependent on drugs to overcome these problems. Therefore, biomechanical spinal manipulations can be used as adjuvant therapy in the treatment of patients suffering from functional scoliosis with hypertension. This therapy can also reduce drug dependence and dosage, thus preventing associated complications and side effects. This trial also reported that participants commencing intervention considered it to be a credible approach. Participants were confident that the spinal manipulations would help them more effectively. Participants who completed the intervention process reported a strong working relationship with the physiotherapist and overall were satisfied with the treatment.

This trial had several notable strengths. Firstly, it was a single-blinded trial and we looked carefully to avoid any bias related to medication and treatment processes that could alter the results. Secondly, we achieved a $100 \%$ retention rate and a $96.67 \%$ adherence rate. At this rate, a fully powered randomized controlled trial was feasible and acceptable for recruiting participants to the main trial. This success rate could have been due to the scheduling of treatment sessions to the patient's convenience.

However, this trial also had some limitations. Firstly, we only included cardiopulmonary parameters in this trial. There were other parameters like spinal alignment (Cobb's angle) and psychological parameters (anxiety and depression) that are affected by spinal curvature.

The data given by this pilot trial will allow to design further randomized control trials to explore it most efficiently. We will also include more outcome measures like spinal alignment (Cobb's angle) and psychological parameters (anxiety and depression) in a fully powered trial.

\section{Conclusion}

The result of this pilot study suggests that it is feasible and acceptable to do a fully powered randomized control trial to assess the effectiveness of spinal manipulations in patients with functional scoliosis. This study also concluded that spinal manipulations combined with Schroth exercises may significantly reduce the SBP and DBP as well as SpO2 levels in patients of with functional scoliosis with hypertension.

\section{Funding}

This research received no external funding.

\section{Conflict of Interest}

The authors have no conflict of interest to declare.

\section{References}

1. Kendall HO, Kendall FP. Developing and Maintaining good Posture. Phys Ther. 1968; 48(4): 319-36.

2. Ruivo RM, Pezarat-Correia P, Carita AI. Cervical and shoulder postural assessment of adolescents between 15 and 17 years old and association with upper quadrant pain. Braz J PhysTher. 2014; 18(4): 364-71.

3. Czaprowski D, Stoliński L, Tyrakowski M, Kozinoga M, Kotwicki T. Non-structural misalignments of body posture in the sagittal plane. Scoliosis Spinal Disord. 2018; 13(6): 1-14.

4. Janani R, Ramachandran A, Divya S. Analysis of Prevalence and Factors Influencing Adolescent Idiopathic Scoliosis Among School Students in Thiruvallur District. Int J Physiother 2019; 6(3): 89-94.

5. Schreiber S, Parent EC, Moez EK, Hedden DM, Hill DL, Moreau M. Schroth Physiotherapeutic ScoliosisSpecific Exercises Added to the Standard of Care Lead to Better Cobb Angle Outcomes in Adolescents with Idiopathic Scoliosis - an Assessor and Statistician Blinded Randomized Controlled Trial. Plos one. 2016: $1-17$.

6. Kotwal S, Pumberger M, Hughes A, Girardi F. Degenerative Scoliosis: A Review. HSS J 2011; 7(3): 257-64.

7. Minghelli B. Scoliosis in adolescence - A revision. Austin Spine. 2017; 1(1): 1002.

8. Sheha ED, Steinhaus ME, Kim HJ, Cunningham ME, Fragomen AT, Rozbruch SR. Leg-Length Discrepancy, Functional Scoliosis, and Low Back Pain. JBJS Reviews. 2018; 6(8): 1-8.

9. Kim DJ, Cho ML, Park YH, Yang YA. Psychological and Physical Effects of Schroth and Pilates Exercise on Female High School Students with Idiopathic Scoliosis. J Phys Ther Sci. 2015; 27(6): 1791-4.

10. Shneerson JM. Pulmonary artery pressure in thoracic scoliosis during and after exercise while breathing air and pure oxygen. Thorax. 1978; 33: 747-54.

11. Huh S, Eun LY, Kim NK, Jung JW, Choi JY, Kim HS. Cardiopulmonary function and scoliosis severity in idiopathic scoliosis children. Korean J Pediatr. 2015; 58(6): 218-23.

12. Gera C, Malik M, Kaur J, Saini M. A systematic review and Meta analysis on effect of spinal mobilization and manipulation on cardiovascular responses. Hong Kong Physiother J. 2020; 40(2): 1-13. 
13. Uneri A, Polat S, Aydingoz O, Bursali A, Migraine vestibulopathy in three families with idiopathic scoliosis: a case series. Cases J. 2009; 2(9367): 1-4

14. Wirth B, Potto T, Rosser S, Humphreys BK. Physical risk factors for adolescent neck and mid back pain: A systematic review. Chiropr Man Ther. 2018; 28(36): $1-10$.

15. Moslehi M, Saiiari A, Marashiyan F. Study of the relationship between Kyphosis, anxiety, depression and aggression of high school boy students. Procedia Soc Behav Sci. 2011; (15): 1798-801.

16. Wang H, Li T, Yuan W, Zhang Z, Wei J, Qiu G, Shen J. Mental health of patients with adolescent idiopathic scoliosis and their parents in China: a cross-sectional survey. BMC Psychiatry. 2019; 19(147): 1-8.

17. Negrini S, Donzelli S, Aulisa AG, Czaprowski D, Schreiber S, Mauroy JCD, et al. 2016 SOSORT guidelines: orthopaedic and rehabilitation treatment of idiopathic scoliosis during growth. Scoliosis Spinal Disord. 2018; 13(3): 1-48.

18. Shah S, Patil P. Effect of posture correction and moderate-intensity exercises on respiratory system in teenagers. Indian J Med Sci. 2019; 71(3): 113-7.

19. Wilkes C, Kydd R, Sagar M, Broadbent E. Upright posture improves affect and fatigue in people with depressive symptoms J Behav Ther Exp Psychiat. 2015; (54): 143-9.

20. Mathersul DC, Rosenbaum S. The Roles of Exercise and Yoga in Ameliorating Depression as a Risk Factor for Cognitive Decline. Evid Based Complement Alternat Med. 2016; 20: 1-9.

21. Chen KM, Kuo CC, Chang YH, Huang HT, Cheng YY. Resistance Band Exercises Reduce Depression and Behavioral Problems of Wheelchair-Bound Older Adults with Dementia: A Cluster-Randomized Controlled Trial. J Am Geriatr Soc. 2017; 65(2): 356-63.

22. Magee DJ. Orthopaedic Physical Assessment. 4th ed. Oxford: Elsevier; 2014.

23. Losa-Iglesias ME, Becerro-de-Bengoa-Vallejo R, Becerro-de-Bengoa-Losa KR. Reliability and concurrent validity of a peripheral pulse oximeter and health-app system for the quantification of heart rate in healthy adults. Health Informatics J. 2014: 1-9.

24. Pérgola PE, White CL, Graves JW, Coffey CS, Tonarelli SB, Hart RG, Benavente OR. Reliability and validity of blood pressure measurement in the Secondary Prevention of Small Subcortical Strokes study. Blood Press Monit. 2007; 12(1): 1-8.
25. Schreiber S, Parent EC, Hill DL, Hedden DM, Moreau MJ, Southon SC. Patients with adolescent idiopathic scoliosis perceive positive improvements regardless of change in the Cobb angle - Results from a randomized controlled trial comparing a 6-month Schroth intervention added to standard care and standard care alone. SOSORT 2018 Award winner. BMC Musculoskelet Disord. 2019; 20(319): 1-10.

26. Thabane L, Ma J, Chu R, Cheng J, Ismaila A, Rios LP, et al. A tutorial on pilot studies: the what, why and how. BMC Med Res Methodol. 2010; 10: 1.

27. Horvath AO, Greenberg LS. Development and validation of the Working Alliance Inventory. J Counselling Psychol. 1989; 36: 223-33.

28. Siljander M, McQuivey K, Gandhi S, Knott P. The Effect of Patient Positioning During Radiographs on The Resulting Cobb Angle Measurements. Orthop Rheumatol Open Access J. 2017; 7(1): 001-4.

29. Bulut MD, Alpayci M, Senkoy E, Bora A, Yazmalar L. Decreased Vertebral Artery Hemodynamics in Patients with Loss of Cervical Lordosis. Med Sci Monit. 2016; 22: 495-500.

30. Wen H, Wang L. Reducing effect of aerobic exercise on blood pressure of essential hypertensive patients. A meta-analysis. Medicine (Baltimore). 2017; 96(11): 1-6.

31. Espí-López GV, Lopez-Bueno L, Vicente-Herrero MT, Arnau FMM, Monzani L. Efficacy of manual therapy on anxiety and depression in patients with tension-type headache. A randomized controlled clinical trial. Int J Osteopath Med. 2016; 22:11-20

32. Masson SC, Tejani AM. Minimum clinically important differences identified for commonly used depression rating scales. J Clinical Epidemiol. 2013; 66(7): 805-7.

33. Charan J, Biswas T. How to calculate sample size for different study designs in medical research? Indian J Psychol Med. 2013; 35(2): 121-6.

34. Tang J, Mandrusiak A, Russell T. The Feasibility and Validity of a Remote Pulse Oximetry System for Pulmonary Rehabilitation: A Pilot Study. Int J Telemed App. 2012: 1-8.

35. Charrois TL, McAlister FA, Dale Cooney D, Lewanczuk R, Kolber MR, Campbell NRC, et al. Improving hypertension management through pharmacist prescribing; the rural alberta clinical trial in optimizing hypertension (Rural RxACTION): trial design and methods. Implement Sci. 2011; 6(94): 1-9. 